\title{
Direct integrability for state feedback optimal control with singular solutions
}

\author{
Paolo Di Giamberardino ${ }^{1}$ and Daniela Iacoviello ${ }^{2}$ \\ 1 Dept. Computer, Control and Management Engineering Antonio Ruberti, Sapienza \\ University of Rome, Italy \\ paolo.digiamberardino@uniroma1.it, \\ WWW home page: http:www.diag.uniroma1.it/ digiamb/webpage.html \\ 2 Dept. Computer, Control and Management Engineering Antonio Ruberti, Sapienza \\ University of Rome, Italy \\ Institute for Systems Analysis and Computer Science Antonio Ruberti, Rome, Italy \\ daniela.iacoviello@uniroma1.it \\ WWW home page: http:www.diag.uniroma1.it/ iacoviel/
}

\begin{abstract}
The paper studies the problem of determining the optimal control when singular arcs are present in the solution. In the general classical approach, the expressions obtained depend on the state and the costate variables at the same time, so requiring a forward-backward integration for the computation of the control. In this paper, firsty sufficient conditions on the dynamics structure are discussed, in order to have both the control and the switching function depending on the state only, computable by a simple forward integration. Then, the possibility to extend this result by means of a preliminary dynamic extension is presented. The approach has been checked and validated making use of a classical SIR epidemic model.
\end{abstract}

Keywords: Optimal Control, Singular Control, Costate Independent Singular Surface, SIR Epidemic Model

\section{Introduction}

Optimal control theory provides the natural framework to solve control problems when contrasting goals are required with resource limitations. The design procedure can make use of the minimum principle, allowing the determination of the optimal control that, depending on the cost index and on the model, could be a bang-bang or a bang-singular-bang solution, [1-4]. In particular, if the model as well as the cost index are linear in the control, the existence conditions of such kind of solutions can be explicitly established.

In the bang-bang solution the control assumes only the extreme values, whereas the singular one is obtained if the Hamiltonian does not depend on the control in an interval of positive measure. The extreme values assumed by the control depend on the sign of the switching function, whereas the existence of singular control is related to the possibility that this switching function is identically zero on an interval of finite length. 
The determination of singular control, while it is easy from a theoretical point of view, is generally difficult to implement; the optimal control requires the solution of a non linear differential equations system in the state variables with initial conditions, and a non linear differential equations system in the costate variables with final conditions. Moreover, in general it is not easy the determination of the best control sequence and the number of switching points, $[5,6]$.

In this paper, the determination of the optimal control of nonlinear systems is investigated referring to the case in which the input acts linearly both in the model and in the cost index, aiming at a constructive computing of the singular solution.

The effectiveness of the approach is tested making use of the example of a classical SIR epidemic model, where S stands for the class of susceptible subjects, I for the class of infected patients and $\mathrm{R}$ for the class of the removed ones $[7,8]$.

Facing epidemic spread control in the framework of optimal control theory is rather common for its capability of suggesting suitable scheduling of possible actions such as vaccination or quarantine or treatment, taking into account resource limitations. Then, it is widely used in literature $[9,10]$.

In particular, the optimal singular control for a SIR epidemic model has been already studied in [11,12], where the structure of singular control has been deeply investigated in presence of the double control, vaccination and medical treatment, showing that the latter can't be singular, whereas a singular regimen is expected for the optimal vaccination strategy.

In this work, the SIR model is used with two different formulations. In a first example, it is assumed that a recovered subject cannot be infected again, while in a second example, the modified case in which the possibility for a recovered individuals to become susceptible again is used. The two formulations allow to deal with different dynamical structures so that all the results presented can be illustrated.

The paper is organized as follows; the classical optimal control problem formulation is given in Section 2; the conditions and the constraints usually considered are illustrated and the structure of the optimal solution, as well as the conditions for the existence of both switches and singular arcs, is given. Then, in Section 3, a particular class of dynamics is introduced, the same considered in [13], and constructive conditions for the singular solutions are given for different formulations of the optimal problem. The example of a SIR model is considered, to put in evidence the procedure and the effectiveness of the results. In Section 4 , the extension of the results to generic dynamics is discussed and it is explicitly formulated for the simpler case of one dimensional dynamic extension. Also in this case, a SIR model in a modified version of the one considered in the previous Section, is considered to exemplify the approach and verify its effectiveness. 


\section{The optimal control problem formulation}

The optimal control problem here addressed is referred to the design of the control input $u$, for a given non linear dynamics of the form

$$
\dot{x}=f(x)+g(x) u=f(x)+\sum_{i=1}^{m} u_{i} g_{i}(x)
$$

where $x \in R^{n}, u \in R^{m}$ and with $x\left(t_{0}\right)=x_{0}$, such that a cost index

$$
J=\int_{t_{0}}^{t_{f}} L(x, u, t) d t
$$

$L: R^{n} \times R^{m} \times R \rightarrow R$ is minimised.

The presence of a bound on the control amplitude can be expressed in the form af a box constraint as

$$
u_{\text {min }} \leq u(t) \leq u_{M A X} \quad \forall t \in\left[t_{0}, t_{f}\right]
$$

with $u_{\min }, u_{M A X} \in R^{m}$.

In the definition of the problem, additional constraints can be introduced. The most common are on (some components of) the final state $x\left(t_{f}\right)$ and on the final time $t_{f}$. Such constraints are considered introducing the function

$$
\chi\left(x\left(t_{f}\right), t_{f}\right)=0
$$

with $\chi \in C^{1}$ almost everywhere, $\operatorname{dim}\{\chi\}=\sigma, 1 \leq \sigma \leq n+1$.

Clearly, if $t_{f}$ is left free, its value has to be found during the optimal control problem solution. In the same way, if some or all the final values for the state components are not prefixed, they are obtained from the problem solution.

The usual design procedure starts with the definition of the Hamiltonian function

$$
H(x, \lambda, u, t)=L(x, u, t)+\lambda^{T}(f(x)+g(x) u)
$$

in which the multiplier function $\lambda(t)$, also called costate, is introduced, with $\lambda(t): R \rightarrow R^{n}, \lambda(t) \in C^{1}$ almost everywhere.

The Hamiltonian (5) verifies the condition

$$
H(x, \lambda, u, t)=K \quad \forall t \in\left[t_{0}, t_{f}\right]
$$

with $K \in R$. Since (6) must satisfy

$$
H\left(x, \lambda, u, t_{f}\right)=\frac{\partial \chi\left(x\left(t_{f}\right), t_{f}\right)}{\partial t_{f}} \eta
$$

for an unknown $\eta \in R, K$ is a variable to be found during the optimization procedure if the final time $t_{f}$ is fixed, with $K=\eta$, while it is equal to zero when the final time is left free and the function $\chi$ does not depends on $t_{f}$. 
From (5), and taking into account the dynamics (1), the costate $\lambda$ must satisfy the differential equation

$$
\dot{\lambda}^{T}=-\frac{\partial H}{\partial x}=-\frac{\partial L}{\partial x}-\lambda^{T}\left(\frac{\partial f}{\partial x}+\sum_{i=1}^{m} u_{i} \frac{\partial g_{i}}{\partial x}\right)
$$

under the boundary conditions

$$
\lambda\left(t_{f}\right)=-\left.\frac{\partial \chi\left(x\left(t_{f}\right), t_{f}\right)}{\partial x\left(t_{f}\right)}\right|^{T} \zeta
$$

with $\zeta \in R^{\sigma}$ to be found and $\chi$ in (4). Clearly, if the final state is unconstrained, (4) does not depends on $x\left(t_{f}\right)$ and (9) becomes

$$
\lambda\left(t_{f}\right)=0
$$

The structure of $(5)$ suggests that if in $L(x, u, t)$ the control $u$ appears linearly, the whole Hamiltonian results to be affine with respect to the control. This property can be very useful when the minimum principle is applied, and it will be used in this paper. Then,

$$
L(x, u, t)=\tilde{L}(x, t)+c^{T} u
$$

can be assumed and, consequently, the expression for (5) becomes

$$
\begin{aligned}
& H(x, \lambda, u, t)=\tilde{L}(x, t)+c^{T} u+\lambda^{T} f(x)+\lambda^{T} g(x) u= \\
& \tilde{L}(x, t)+\lambda^{T} f(x)+\left(c^{T}+\lambda^{T} g(x)\right) u=F(x, \lambda, t)+G(x, \lambda) u
\end{aligned}
$$

with

$$
\begin{aligned}
F(x, \lambda, t) & =\tilde{L}(x, t)+\lambda^{T} f(x) \\
G(x, \lambda) & =c^{T}+\lambda^{T} g(x)
\end{aligned}
$$

Clearly, the presence in the cost function of the input $u$ in a linear form is always possible if $u_{\min } \geq 0$ in (3).

The minimum principle

$$
H(x, \lambda, u, t) \leq H(x, \lambda, \omega, t) \quad \forall \omega \in\left[u_{\min }, u_{M A X}\right]
$$

can be used, yielding, for (12), the condition

$$
G(x, \lambda) u \leq G(x, \lambda) \omega \quad \forall \omega \in\left[u_{\min }, u_{M A X}\right]
$$

As a consequence, the optimal control

$$
u= \begin{cases}u_{M A X} & \text { if } G(x, \lambda)<0 \\ u_{\min } & \text { if } G(x, \lambda)>0\end{cases}
$$


can be obtained. The time instant $t_{s}$ such that

$$
G\left(x\left(t_{s}\right), \lambda\left(t_{s}\right)\right)=0
$$

with

$$
G\left(x\left(t_{s}^{-}\right), \lambda\left(t_{s}^{-}\right)\right) \cdot G\left(x\left(t_{s}^{+}\right), \lambda\left(t_{s}^{+}\right)\right)<0
$$

is the instant of switching in which the control changes from one extreme to the other, so getting a classical bang-bang solution.

If condition (18) holds over a finite time interval $\left[t_{1}, t_{2}\right] \subseteq\left[t_{i}, t_{f}\right]$

$$
G(x(t), \lambda(t))=0 \quad t \in\left[t_{1}, t_{2}\right]
$$

then the optimal control presents a singular arc in the solution and it assumes the expression

$$
u= \begin{cases}u_{M A X} & \text { if } G(x, \lambda)<0 \\ u_{s}(x, \lambda) & \text { if } G(x, \lambda)=0 \\ u_{\min } & \text { if } G(x, \lambda)>0\end{cases}
$$

In this case, (19) means that $G(x(t), \lambda(t))$ is constant over a finite time interval and, then, all its time derivatives in the same interval must be equal to zero; so, the identities

$$
\frac{\partial^{k} G(x(t), \lambda(t))}{\partial t^{k}} \doteq G^{(k)}(x(t), \lambda(t))=0 \quad \forall t \in\left[t_{1}, t_{2}\right]
$$

must hold for any $k \geq 0$. By definition, $G^{(0)}(x(t), \lambda(t))$ does not depend on the control $u$. Computing (21), for $k=0,1,2, \ldots$, there exists an index $r$, necessarily even $[11,12]$, such that $G^{(k)}(x(t), \lambda(t))$ is independent from $u$ if $k<r$, while, for $k=r$, the control $u$ appears explicitly [4]. Then, the first $r$ conditions (21), for $k=0, \ldots, r-1$, give $r$ relations between state $x$ and costate $\lambda$, and from $G^{(r)}(x(t), \lambda(t))=0$ the expression for the singular control $u_{s}$ in (20) can be obtained.

Making reference to the expression (14), one has

$$
\begin{gathered}
G^{(0)}(x, \lambda)=c^{T}+\lambda^{T} g(x)=0 \\
G^{(1)}(x, \lambda)=\dot{\lambda}^{T} g(x)+\lambda^{T} \dot{g}(x)= \\
-\alpha^{T} g(x)-\lambda^{T} L_{g} f(x)-\sum_{i=1}^{m} u_{i} \lambda^{T} L_{g} g_{i}(x)+\lambda^{T} L_{f} g(x)+\sum_{i=1}^{m} u_{i} \lambda^{T} L_{g_{i}} g(x)= \\
-\alpha^{T} g(x)+\lambda^{T} a d_{f} g(x)+\sum_{i=1}^{m} u_{i} \lambda^{T} a d_{g_{i}} g(x)=0
\end{gathered}
$$


where the compact expressions

$$
\begin{aligned}
L_{f} g(x) & =\left(L_{f} g_{1}(x) \ldots L_{f} g_{m}(x)\right) \\
L_{g} f(x) & =\left(L_{g_{1}} f(x) \ldots L_{g_{m}} f(x)\right) \\
L_{g} g_{i}(x) & =\left(L_{g_{1}} g_{i}(x) \ldots L_{g_{m}} g_{i}(x)\right) \\
L_{g_{i}} g(x) & =\left(L_{g_{i}} g_{1}(x) \ldots L_{g_{i}} g_{m}(x)\right) \\
a d_{f} g(x) & =L_{f} g(x)-L_{g} f(x) \\
a d_{g_{i}} g(x) & =L_{g_{i}} g(x)-L_{g} g_{i}(x)
\end{aligned}
$$

are used. Note that the structures of the Lie Bracket introduced are

$$
\begin{gathered}
a d_{f} g(x)=\left(a d_{f} g_{1}(x) a d_{f} g_{2}(x) \cdots a d_{f} g_{m}(x)\right) \\
a d_{g_{i}} g(x)=\left(a d_{g_{i}} g_{1}(x) a d_{g_{i}} g_{2}(x) \cdots a d_{g_{i}} g_{m}(x)\right)
\end{gathered}
$$

recalling that $a d_{g_{i}} g_{i}(x)=0 \forall i \in[1, m]$.

If the vector fields $g_{i}$ in (1) commute, then $\sum_{i=1}^{m} u_{i} \lambda^{T} a d_{g_{i}} g(x)=0$, as in [11, $12]$; the same term is equal to zero also if single input systems are considered. In both cases, the identity (23) reduces to

$$
G^{(1)}(x, \lambda)=-\alpha^{T} g+\lambda^{T} a d_{f} g(x)=0
$$

and $G^{(2)}(x, \lambda)$ can be computed using this simplified expression, iterating the procedure.

\section{The case of block sub triangular single input systems}

In this Section, the class of dynamics (1) considered is particularised setting $x$ non negative and $m=1$, as in [13]. This simplification allows to better clarify the relationships between the structure of the system and the possibility to compute the optimal control law avoiding the backward integration of the costate dynamics (8).

Under these hypothesis, the initial dynamics (1) is simplified and (24) holds: moreover, thanks to the non negativeness of the state,

$$
\tilde{L}(x, t)=\alpha^{T} x
$$

can be used, so obtaining a fully linear term in the cost function

$$
L(x, u, t)=L(x, u)=\alpha^{T} x+c u
$$

Then, $\dot{\lambda}^{T}$ in (8) becomes

$$
\dot{\lambda}^{T}=-\alpha^{T}-\lambda^{T} \frac{\partial f}{\partial x}-u \lambda^{T} \frac{\partial g}{\partial x}
$$


and the expressions of (22) and (23) become

$$
\begin{aligned}
& G^{(0)}(x, \lambda)=c+\lambda^{T} g(x)=0 \\
& G^{(1)}(x, \lambda)=-\alpha^{T} g(x)+\lambda^{T} a d_{f} g(x)=0
\end{aligned}
$$

with (29) always independent from $u$. Then, the computation of $G^{(2)}(x, \lambda, u)=0$ must always be performed:

$$
\begin{aligned}
& G^{(2)}(x, \lambda, u)=-\alpha^{T}\left(L_{f} g(x)+u L_{g} g(x)\right) \\
& -\left(\alpha^{T}+\lambda^{T} \frac{\partial f}{\partial x}+u \lambda^{T} \frac{\partial g}{\partial x}\right) a d_{f} g(x)+\lambda^{T}\left(L_{f} a d_{f} g(x)+u L_{g} a d_{f} g(x)\right)= \\
& \left(\lambda^{T} a d_{f}^{2} g(x)-\alpha^{T}\left(L_{f} g(x)+a d_{f} g(x)\right)\right)-\left(\alpha^{T} L_{g} g(x)-\lambda^{T} a d_{g} a d_{f} g(x)\right) u
\end{aligned}
$$

If $\alpha^{T} L_{g} g(x)-\lambda^{T} a d_{g} a d_{f} g(x) \neq 0$, the singular control $u_{s}$ can be obtained as

$$
u_{s}(x, \lambda)=\frac{\lambda^{T} a d_{f}^{2} g(x)-\alpha^{T}\left(L_{f} g(x)+a d_{f} g(x)\right)}{\alpha^{T} L_{g} g(x)-\lambda^{T} a d_{g} a d_{f} g(x)}
$$

otherwise $G^{(2)}(x, \lambda, u)=G^{(2)}(x, \lambda)$, the condition

$$
\lambda^{T} a d_{f}^{2} g(x)-\alpha^{T}\left(L_{f} g(x)+a d_{f} g(x)\right)=0
$$

involving $x$ and $\lambda$ only, is obtained, and a further derivative $G^{(3)}(x, \lambda, u)$ must be computed. Set $r$ as the first index such that $G^{(r)}(x, \lambda, u)$ is dependent from the input. It is easily verified that:

Proposition 1. The expression of the $i-$ th derivative $G^{(i)}(x, \lambda)$, for $i=0,1, \ldots, r-$ 1 , is of the form

$$
G^{(i)}(x, \lambda)=\lambda^{T} a d_{f}^{i} g(x)+h_{i}(x)
$$

for suitable functions $h_{i}(x)\left(h_{0}(x)=c, h_{1}(x)=-\alpha^{T} g(x)\right)$.

Proof. It comes iteratively at the $i$-th step from the structure of $G^{(i-1)}(x, \lambda)$ and the computations evidenced in (30).

\subsection{The class of dynamics considered}

The class of nonlinear dynamics considered in this Section presents the structure

$$
\left(\begin{array}{c}
\dot{x}_{1} \\
\dot{x}_{2}
\end{array}\right)=\left(\begin{array}{c}
f_{1}\left(x_{1}\right) \\
f_{2}\left(x_{1}\right)
\end{array}\right)+\left(\begin{array}{c}
g_{1}\left(x_{1}\right) \\
g_{2}\left(x_{1}\right)
\end{array}\right) u
$$

where $x_{1} \in R^{r}, x_{2} \in R^{n-r}$, and the functions $f_{i}$ and $g_{i}$ defined consequently. The controllability condition

$$
\operatorname{dim}\left(\operatorname{span}\left\{g_{1}, a d_{f_{1}} g_{1}, \ldots, a d_{f_{1}}^{r-1} g\right\}\left(x_{1}\right)\right)=r
$$


for the first subsystem is assumed verified, with $x_{1}$ in a suitable domain containing $x_{1}\left(t_{0}\right)$.

In order to avoid long computations, the simplest case of $r=2$ is here addressed, for which all the expressions can be written in a compact form. This is also the case of the example discussed in Subsection 3.3 to illustrate the proposed approach.

Consequently, setting

$$
\lambda=\left(\begin{array}{c}
\lambda_{1} \\
\lambda_{2}
\end{array}\right) \quad \lambda_{1} \in R^{r}, \lambda_{2} \in R^{n-r}
$$

one has

$$
\begin{aligned}
& \frac{\partial L}{\partial x}=\left(\frac{\partial L}{\partial x_{1}} \frac{\partial L}{\partial x_{2}}\right) \\
& \frac{\partial f}{\partial x}=\left(\begin{array}{c}
\frac{\partial f_{1}\left(x_{1}\right)}{\partial x_{1}} 0 \\
\frac{\partial f_{2}\left(x_{1}\right)}{\partial x_{1}} 0
\end{array}\right) \quad \frac{\partial g}{\partial x}=\left(\begin{array}{c}
\frac{\partial g_{1}\left(x_{1}\right)}{\partial x_{1}} 0 \\
\frac{\partial g_{2}\left(x_{1}\right)}{\partial x_{1}} 0
\end{array}\right)
\end{aligned}
$$

so that (8) becomes

$$
\begin{aligned}
& \left(\begin{array}{l}
\dot{\lambda}_{1} \\
\dot{\lambda}_{2}
\end{array}\right)=-\left(\begin{array}{c}
\frac{\partial L}{\partial x_{1}} \\
\frac{\partial L}{\partial x_{2}}
\end{array}\right)+\left(\begin{array}{cc}
\frac{\partial f_{1}^{T}\left(x_{1}\right)}{\partial x_{1}} & \frac{\partial f_{2}^{T}\left(x_{1}\right)}{\partial x_{1}} \\
0 & 0
\end{array}\right) \lambda+ \\
& +u\left(\begin{array}{cc}
\frac{\partial g_{1}\left(x_{1}\right)}{\partial x_{1}} & \frac{\partial g_{2}\left(x_{1}\right)}{\partial x_{1}} \\
0 & 0
\end{array}\right) \lambda
\end{aligned}
$$

The above equation can be explicitly decomposed into

$$
\begin{aligned}
\dot{\lambda}_{1}= & -\frac{\partial L}{\partial x_{1}}-\left(\frac{\partial f_{1}^{T}\left(x_{1}\right)}{\partial x_{1}}+u \frac{\partial g_{1}\left(x_{1}\right)}{\partial x_{1}}\right) \lambda_{1}+ \\
& -\left(\frac{\partial f_{2}^{T}\left(x_{1}\right)}{\partial x_{1}}+u \frac{\partial g_{2}\left(x_{1}\right)}{\partial x_{1}}\right) \lambda_{2} \\
\dot{\lambda}_{2}= & -\frac{\partial L}{\partial x_{2}}
\end{aligned}
$$

\subsection{Results for the class of dynamics considered}

The particular structure of the system (33), which induces the simplification on the $\lambda$ dynamics (37)-(38), has been used in [13] to prove the following results, for which a short justification is here recalled, mainly focusing on the computation of the costate. The differences among the following three Proposition are in the boundary conditions, studying the differences for fixed or free final time and/or state.

Proposition 2. Consider the optimal control problem, with fixed final time $t_{f}$ and free final conditions on the state, for a non linear dynamics of the form 
(33) and the cost function (2) with $L$ as in (26). There exists a procedure for the computation of the optimal singular solution where the control law is as a pure state feedback, bringing to a bang-singular-bang optimal control, for which the singular manifold can be explicitly given as a state function only. Moreover, after the last switch, the optimal control $u$ is equal to $u_{\text {min }}$.

Proof. [13]: under the conditions stated in the Proposition, the constraint (4) does not depend on the final state $x\left(t_{f}\right)$, so that (10) holds; the expression of $L(x, u, t)$ gives $\dot{\lambda}_{2}=0$ in (38) and, thanks to (10) and the continuity hypothesis on $\lambda(t)$, one has

$$
\lambda_{2}(t)=0 \quad \forall t \in\left[t_{0}, t_{f}\right]
$$

As far as $\lambda_{1}$ is concerned, equations $G^{(i)}(x, \lambda), i=0,1, \ldots, r-1$, can be written, for Proposition 2, as

$$
\begin{aligned}
G^{(0)}(x, \lambda) & =\lambda_{1}^{T} g_{1}\left(x_{1}\right)+c=0 \\
G^{(1)}(x, \lambda) & =\lambda_{1}^{T} a d_{f_{1}} g_{1}\left(x_{1}\right)-\alpha_{1}^{T} g_{1}\left(x_{1}\right)=0 \\
\cdots & =\ldots \\
G^{(r-1)}(x, \lambda) & =\lambda_{1}^{T} a d_{f_{1}}^{r-1} g_{1}\left(x_{1}\right)+h_{r-1}\left(x_{1}\right)=0
\end{aligned}
$$

Rewritten in the compact form

$$
\begin{gathered}
\lambda_{1}^{T}\left(g_{1}\left(x_{1}\right), a d_{f_{1}} g_{1}\left(x_{1}\right), \ldots a d_{f_{1}}^{i-1} g_{1}\left(x_{1}\right)\right)= \\
=\left(-c, \alpha_{1}^{T} g_{1}\left(x_{1}\right), \ldots h_{i-1}\left(x_{1}\right)\right)
\end{gathered}
$$

the expression of $\lambda_{1}$

$$
\lambda_{1}^{T}=\left(-c, \ldots h_{i-1}\left(x_{1}\right)\right)\left(g_{1}\left(x_{1}\right), \ldots a d_{f_{1}}^{i-1} g_{1}\left(x_{1}\right)\right)^{-1}
$$

can be easily computed once (34) holds. Then, the expression of the costate $\lambda$ is fully known, $\lambda_{2}(t)$ as in (39) and $\lambda_{1}$ as in (43). It is a function of the state, without the necessity of using (36), so avoiding the backward integration procedure.

Then, it is possible to express the singular control $u_{s}(x, \lambda)$ as a state function only, in particular a function of $x_{1}, u_{s}=u_{s}\left(x_{1}\right)$.

The equation of the singular manifold can be obtained from

$$
F(x, \lambda, t)=\alpha_{1}^{T} x_{1}+\lambda_{1}^{T} f_{1}\left(x_{1}\right)=K
$$

thanks to (6), in which the structure (12) is used, and the fact that condition (19) holds. Expression (44) can be explicitly written as a function of the state once (43) is used:

$$
K=\alpha_{1}^{T} x_{1}+\left(-c \ldots h_{i-1}\left(x_{1}\right)\right)\left(g_{1}\left(x_{1}\right) \ldots a d_{f_{1}}^{i-1} g_{1}\left(x_{1}\right)\right)^{-1} f_{1}\left(x_{1}\right)
$$


which fully describes the singular surface in the $x_{1}$ subspace. The subspace $x_{2}$ can be computed from $x_{1}$, thanks to the structure of (33). The value of the unknown parameter $K$ can be obtained noting that, from (12) evaluated af $t=t_{f}$, one has

$$
H\left(x, \lambda, u, t_{f}\right)=\alpha_{1}^{T} x_{1}\left(t_{f}\right)=K
$$

being $u\left(t_{f}\right)=0$ as well as $\lambda\left(t_{f}\right)=0$. This procedure allows to fully compute the singular part of the optimal solution. The full solution (20) is obtained including the conditions in (17).

Other two Propositions, shortly recalled below, are proved in [13], where the same problem as in Proposition 2 is defined but with different conditions on the final state and time.

More explicitly, while Proposition 2 refers to the case of fixed final time $t_{f}$ and free final state $x\left(t_{f}\right)$, Proposition 3 considers both $x\left(t_{f}\right)$ and the time $t_{f}$ fixed, and Proposition 4 refers to fixed final state and free final time. All these choices bring to different definitions of the constraint function (4) which determines the boundary conditions on $\lambda(t)$ and on (5). The core of the results, the possibility to have the expression for $\lambda(t)$ directly as a function of the state without its differential system integration, is the same for all the three cases.

Proposition 3. Given the optimal control problem, with fixed time $t_{f}$ and fixed final condition on the state $x\left(t_{f}\right)=x_{f}$, for a non linear dynamics of the form (33) and the cost function (2) with $L$ of the form (26), there exists a procedure for the computation of the optimal singular solution which gives the expression of the control law as a pure state feedback, bringing to a bang-singular-bang optimal control, for which the singular surface can be explicitly written as a state function only.

In this case, the presence of constraint (4) does not allow to know $\lambda\left(t_{f}\right)$ due to expression (9). However, $\dot{\lambda}_{2}=0$ is still true and then one has $\lambda_{2}(t)=\lambda_{2}\left(t_{f}\right)=$ const. In this case equations (40), (41), (42) assume the form

$$
\begin{aligned}
G^{(0)}(x, \lambda) & =\lambda_{1}^{T} g_{1}\left(x_{1}\right)+\lambda_{2}^{T}\left(t_{f}\right) g_{2}\left(x_{1}\right)=0 \\
G^{(1)}(x, \lambda) & =\lambda_{1}^{T} a d_{f_{1}} g_{1}\left(x_{1}\right)-\alpha_{1}^{T} g_{1}\left(x_{1}\right)+\lambda_{2}^{T}\left(t_{f}\right)\left[f_{2}, g_{2}\right]\left(x_{1}\right)=0 \\
\ldots & =\ldots \\
G^{(r-1)}(x, \lambda) & =\lambda_{1}^{T} a d_{f_{1}}^{r-1} g_{1}\left(x_{1}\right)+h_{r-1}\left(\lambda_{2}\left(t_{f}\right), x_{1}\right)
\end{aligned}
$$

Following the same considerations as in the previous Proposition 2, it is possible to compute $\lambda_{1}$ as in (43), whose expression in this case is $\lambda_{1}\left(\lambda_{2}\left(t_{f}\right), x_{1}\right)$. Also the singular control $u_{s}$ as weel as the singular surface (45) can be computed as function of $x_{1}$, but they results to be parametrised by $\lambda_{2}\left(t_{f}\right)$. The same follows for $x_{2}(t)$. However, thanks to condition (4), it is possible to determine the actual values for $\lambda_{2}\left(t_{f}\right)$, so getting again the full computation of the singular solution $u_{s}=u_{s}\left(x_{1}\right)$ and of the singular state space manifold. The remaining part of the procedure follows what stated in Proposition 2.

The last case here addressed refers to a free time fixed final conditions on the state variables. It is possible to state the following 
Proposition 4. Given the optimal control problem, with free final time $t_{f}$ and the constraint (4) for the final conditions on the state, for a non linear dynamics of the form (33) and the cost function (2) with $L(x, u, t)=\alpha_{1}^{T} x_{1}+c u, \alpha_{1} \in R^{r}$, there exists an algorithm for the computation of the optimal singular solution which gives the expression of the control law as a pure state feedback, bringing to a bang-singular-bang optimal control, for which the singular surface can be explicitly written as a state function only.

Proof: The proof comes straightforwardly from the one of Proposition 3, since the only difference is in the fact that being the final time $t_{f}$ free, $K$ in (6) and in all the derived expressions, as far as (45), are equal to zero. All the other considerations still hold.

These results are applied, in next Subsection, to a simple dynamical system in order to put in evidence the main steps and the results.

\subsection{The example of a SIR model}

The effectiveness of the procedures introduced is here verified making use of a classical problem, the optimal control of an epidemic spread. The mathematical model here considered is a SIR dynamics.

With reference to the general formulation introduced in Section 2, the classical model of a SIR epidemic spread is

$$
\begin{aligned}
\dot{S} & =-\beta S I-S u+\mu \\
\dot{I} & =\beta S I-\gamma I \\
\dot{R} & =\gamma I
\end{aligned}
$$

with given initial conditions $S_{0}, I_{0}, R_{0}$, and box constraints $u(t) \in\left[0, U_{M A X}\right]$, for which the control law $u(t)$ minimising the cost function

$$
J(S(t), I(t), R(t), u(t))=\int_{t_{0}}^{t_{f}}(a I(t)+c u(t)) d t
$$

has to be found. Only the final time $t_{f}$ is assumed fixed and equal to $t_{f}^{d}$. Then, the constraint (4) has the expression $\chi\left(t_{f}\right)=t_{f}-t_{f}^{d}$ and its derivative with respect to $t_{f}$ is equal to one. The aim of the control action is to minimize the number of infected subjects $I$ in the fixed time interval $\left[0, t_{f}\right]$ by using as less resources as possible; the positive parameters $a$ and $c$ represent the weights of these two contrasting requirements.

Setting

$$
x=\left(\begin{array}{l}
x_{1} \\
x_{2}
\end{array}\right) \quad \text { with } \quad x_{1}=\left(\begin{array}{l}
S \\
I
\end{array}\right) \quad \text { and } \quad x_{2}=R
$$

the formulation corresponds to the case considered in Proposition 2, with $r=2$, $\alpha_{1}^{T}=\left(\begin{array}{ll}0 & a\end{array}\right), \alpha^{T}=\left(\alpha_{1}^{T} 0\right)$ and 


$$
\begin{array}{ll}
f_{1}\left(x_{1}\right) & =\left(\begin{array}{c}
-\beta S I+\mu \\
\beta S I-\gamma I
\end{array}\right) g_{1}\left(x_{1}\right)=\left(\begin{array}{c}
-S \\
0
\end{array}\right) \\
f_{2}\left(x_{1}\right) & =\gamma I \quad g_{2}\left(x_{1}\right)=0
\end{array}
$$

Correspondingly, the costate $\lambda$ is defined as

$$
\lambda=\left(\begin{array}{l}
\lambda_{1} \\
\lambda_{2}
\end{array}\right) \quad \text { with } \quad \lambda_{1} \in R^{2} \quad \text { and } \quad \lambda_{2} \in R
$$

and the Hamiltonian (12) is

$$
\begin{aligned}
H(x, \lambda, u)= & \alpha_{1}^{T} x_{1}+c u+\lambda_{1}^{T} f_{1}\left(x_{1}\right)+\lambda_{2} f_{2}\left(x_{1}\right)+\lambda_{1}^{T} g_{1}\left(x_{1}\right) u= \\
& F(x, \lambda)+G(x, \lambda) u
\end{aligned}
$$

with

$$
\begin{aligned}
& F(x, \lambda)=\alpha_{1}^{T} x_{1}+\lambda_{1}^{T} f_{1}\left(x_{1}\right)+\lambda_{2} f_{2}\left(x_{1}\right) \\
& G(x, \lambda)=c+\lambda_{1}^{T} g_{1}\left(x_{1}\right)
\end{aligned}
$$

and for which

$$
H(x, \lambda, u)=K \quad \forall t \in\left[t_{0}, t_{f}\right], \quad K \in R
$$

The costate dynamics (37) and (38) for $\lambda$ can be computed, getting

$$
\begin{aligned}
& \dot{\lambda}_{1}=-\left(\begin{array}{l}
0 \\
a
\end{array}\right)-\left(\begin{array}{cc}
-\beta I & \beta I \\
-\beta S & \beta S-\gamma
\end{array}\right) \lambda_{1}-u\left(\begin{array}{cc}
-1 & 0 \\
0 & 0
\end{array}\right) \lambda_{1}-\left(\begin{array}{l}
0 \\
\gamma
\end{array}\right) \lambda_{2} \\
& \dot{\lambda}_{2}=0
\end{aligned}
$$

with $\lambda\left(t_{f}\right)=0$ due to the free final state condition. Then, as expected, from (62) one has $\lambda_{2}(t)=0$.

Performing the computations, expressions (47) and (48) assume the form

$$
\begin{aligned}
& G^{(0)}(x, \lambda)=c+\lambda_{1}^{T}\left(\begin{array}{c}
-S \\
0
\end{array}\right)=0 \\
& G^{(1)}(x, \lambda)=\lambda_{1}^{T}\left(\begin{array}{c}
-\mu \\
\beta S I
\end{array}\right)=0
\end{aligned}
$$

from which the variable $\lambda_{1}$ can be computed, as a function of $x_{1}=\left(\begin{array}{c}S \\ I\end{array}\right)$, obtaining

$$
\lambda_{1}=\left(\begin{array}{cc}
-S & 0 \\
-\mu & \beta S I
\end{array}\right)^{-1}\left(\begin{array}{c}
-c \\
0
\end{array}\right)=\left(\begin{array}{c}
\frac{c}{S} \\
\frac{\mu c}{\beta S^{2} I}
\end{array}\right)
$$

Moreover, for $G^{(2)}(x, \lambda)$ one has

$$
G^{(2)}(x, \lambda)=\lambda_{1}^{T} a d_{f_{1}}^{2} g_{1}\left(x_{1}\right)-\alpha_{1}^{T} a d_{f_{1}} g_{1}\left(x_{1}\right)+u \lambda_{1}^{T} a d_{g_{1}} a d_{f_{1}} g_{1}\left(x_{1}\right)
$$


from which the singular control

$$
u_{s}(x, \lambda)=\frac{\lambda_{1}^{T} a d_{f_{1}}^{2} g_{1}\left(x_{1}\right)-\alpha_{1}^{T} a d_{f_{1}} g_{1}\left(x_{1}\right)}{\lambda_{1}^{T} a d_{g_{1}} a d_{f_{1}} g_{1}\left(x_{1}\right)}
$$

is obtained. After all the computations, and making use of (65), the state feedback singular control

$$
u_{s}(x)=\frac{a \beta S I-\beta^{2} c S I+\frac{2 \beta c \mu I}{S}-\frac{2 c \mu^{2}}{S^{2}}}{-2 \frac{c \mu}{S}}=\beta I\left(\frac{\beta c-a}{2 c \mu} S^{2}-1\right)+\frac{\mu}{S}
$$

is computed. The singular manifold can be obtained as a state function according to (44) and (45):

$$
\left(\begin{array}{ll}
0 & a
\end{array}\right)\left(\begin{array}{l}
S \\
I
\end{array}\right)+\left(\begin{array}{ll}
\frac{c}{S} & \frac{\mu c}{\beta S^{2} I}
\end{array}\right)\left(\begin{array}{c}
-\beta S I+\mu \\
\beta S I-\gamma I
\end{array}\right)=a I-\beta c I+2 \frac{c \mu}{S}-\frac{c \gamma \mu}{\beta S^{2}}=K
$$

Finally, from (46),

$$
K=a I\left(t_{f}\right)
$$

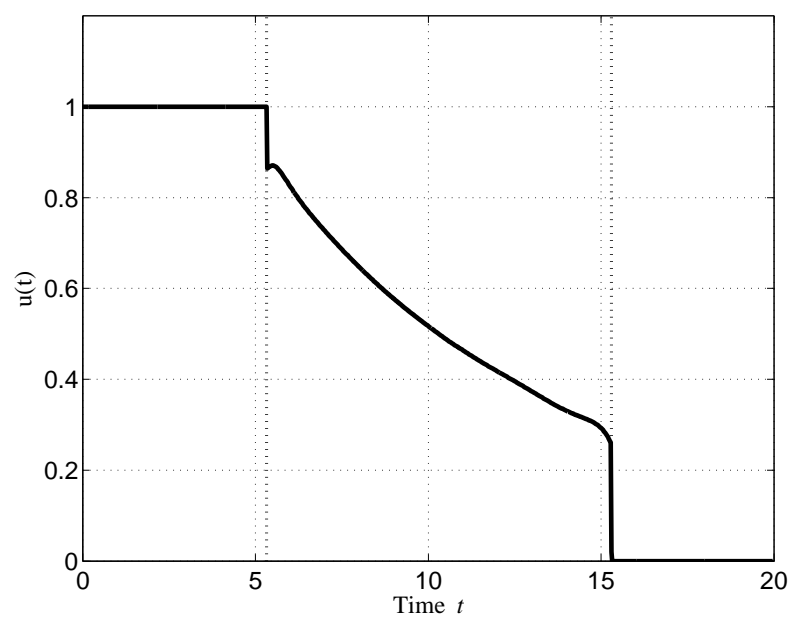

Fig. 1. The Bang-Singular-Bang optimal control $u(t)([13])$.

The simulation has been performed setting $t_{f}=20$, fixing the model parameters to $\beta=0.01, \gamma=0.4$ and $\mu=10$ and choosing the weights $\alpha=1$ and $c=1$ in the cost function (53). The time history of the bang-singular-bang control is depicted in Figure 1, where the finite time interval of the singular solution $t \in\left[t_{1}, t_{2}\right]$, with $t_{1}=5.32$ and $t_{2}=15.3$, is evidenced by the two vertical dotted 


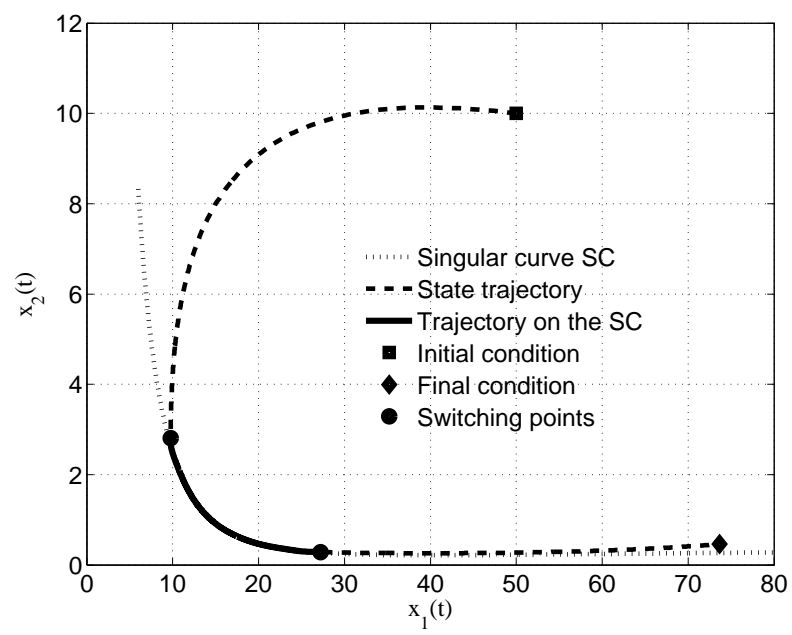

Fig. 2. State trajectory in the $x_{1}-x_{2}$ plane, compared with the singular curve $([13])$.

lines. The corresponding state evolution is represented in Figure 2 for the $x_{1}-x_{2}$ plane projection: the initial part of the trajectory from the starting conditions $x_{1,0}=50$ and $x_{2,0}=10$, the squared point, to the intersection with the singular curve (dotted line), denoted by a dashed curve, represents the evolution under the upper bound value for the control. Then, under the singular control (68), the trajectory follows the singular curve along the solid arc, until the second switch from $u=u_{s}$ to $u=u_{\min }=0$ occurs, yielding to the free evolution from the singular arc to the final point (diamond marker).

\section{An extended case}

The results presented in Section 3 are based on the particular form for dynamics (33) and its relationship with condition (34).

The use of these results on a generic system can be extended once it is observed that if in the dynamics (33) one has $r=n$, the expression of a generic $n$ dimensional system is obtained which has to satisfy the full controllability condition (34). For such a system, the procedure described above can be easily applied. In fact, in this case, the full costate can be obtained as a function of the state making use of (43) with $r=n$. Then, the procedures described in Proposition 2, Proposition 3 and Proposition 4 can be applied and the optimal control problem can be solved without requiring the solution, by backward integration, of the differential system (27) and yielding to an optimal control law that can be expressed as a state function, that is in the form of a state feedback.

The fulfilment of condition $r=n$ is not common, being impossible if $n$ is odd. So, also the direct use of the results in Section 3 is strongly limited. 
In this Section, the possibility to extend the results to the case of generic dynamics for which the condition $r<n$ holds is here addressed.

The enriched control scheme is described for the case $r=n-1$, since the other cases can be obtained recursively.

In this case the optimal control problem is referred to a dynamics (1) with $x \in R^{n}, x_{i} \geq 0$, and $u \in R, u \geq 0$, to a cost function (2) with position (25), and control constraints (3) with $u_{\text {min }}=0$.

The introduction of the Hamiltonian function (12) and the use of the minimum principle (15), which becomes (16), allow to obtain the expression (17) for the optimal control.

If a singular arc is present in the optimal solution, then the expressions of the $G^{(i)}(x, \lambda)$ can be computed and, for the hypothesis assumed on $r$, and for the Proposition 1 , one has that for the first $n-1$ terms $G^{(i)}(x, \lambda), i=0, \ldots, n-2$, the following conditions

$$
\begin{aligned}
G^{(0)}(x, \lambda) & =\lambda^{T} g(x)+c=0 \\
G^{(1)}(x, \lambda) & =\lambda^{T} a d_{f} g(x)-\alpha^{T} g(x)=0 \\
\cdots & \cdots \cdots \\
G^{(n-2)}(x, \lambda) & =\lambda^{T} a d_{f}^{n-2} g(x)+h_{n-2}(x)=0
\end{aligned}
$$

hold independently from the control $u$, while, at the $n-1$-th step, one gets the form, as the one in (30)

$$
G^{(n-1)}(x, \lambda, u)=M(x, \lambda)+N(x, \lambda) u=0
$$

with $N(x, \lambda, u) \neq 0$ so that the singular control

$$
u_{s}=-\frac{M(x, \lambda)}{N(x, \lambda)}
$$

can be computed. In this case, without particular hypothesis on the dynamics, from (43) with $r=n-2$, it is not possible to obtain the $n$ components of the costate $\lambda$ from the $n-1$ dimensional system (71).

The approach here followed to overcome this problem makes use of a dynamic extension of the input, adding an integrator on $u$. Then, $u$ becomes one component of the state and $v=\dot{u}$ is the new input. This position has the consequence of making (72) the $(n-1)$-th equation of system (71) from which a solution like (43) can be obtained for the costate $\lambda$.

The drawback of this idea is that the dimension of the state becomes $n+1$ and the same is, consequently, the dimension of the costate. Then, since from (43) only $n$ components of the costate can be computed, it seems that the problem is not actually solved.

In order to clarify the effects of the dynamic extension on the whole optimal control problem, the reformulation for the extended dynamics is performed and the steps for the computation of the new solutions are analysed. 
Then, consider now the extended dynamics

$$
\begin{aligned}
& \dot{x}=f(x)+g(x) u \\
& \dot{u}=v
\end{aligned}
$$

with $x \in R^{n}, u \in R, v \in R$, which can be described by

$$
\dot{z}=\Phi(z)+\Gamma v
$$

once the extended state $z=\left(x^{T} u\right)^{T}$ is introduced, as long as the extended vector fields

$$
\Phi(z)=\left(\begin{array}{c}
f(x)+g(x) u \\
0
\end{array}\right), \quad \Gamma=\left(\begin{array}{l}
0 \\
1
\end{array}\right)
$$

Clearly, the same cost function (2) with (26) is considered, being it the original cost of the problem. Its expression, for the extended case, becomes

$$
J=\int_{t_{0}}^{t_{f}}\left(\alpha^{T} x+c u\right) d t=\int_{t_{0}}^{t_{f}} \tilde{\alpha}^{T} z d t
$$

The external input $v$ is not present in the cost function and is not explicitly constrained, since the previous bound on $u$,

$$
u(t) \in\left[0, u_{M A X}\right] \quad \forall t \in\left[t_{0}, t_{f}\right]
$$

here becomes a constraint on one of the state components.

As far as the boundary conditions on the state, $x\left(t_{0}\right)=x_{0}$ is the same as in the original problem; for $u$, the initial and/or the final values can be assumed known, once the existence of the control (20) is assumed for the initial problem.

Also the constraint (4) must be rewritten with respect to the extended state, getting the expression

$$
\tilde{\chi}\left(z\left(t_{f}\right), t_{f}\right)=0
$$

verifying

$$
\frac{\partial \tilde{\chi}\left(z\left(t_{f}\right), t_{f}\right)}{\partial z\left(t_{f}\right)}=\left(\frac{\partial \tilde{\chi}\left(x\left(t_{f}\right), u\left(t_{f}\right), t_{f}\right)}{\partial x\left(t_{f}\right)} \frac{\partial \tilde{\chi}\left(x\left(t_{f}\right), u\left(t_{f}\right), t_{f}\right)}{\partial u\left(t_{f}\right)}\right)
$$

where

$$
\frac{\partial \tilde{\chi}\left(x\left(t_{f}\right), u\left(t_{f}\right), t_{f}\right)}{\partial u\left(t_{f}\right)}=0
$$

if no hypothesis can be initially formulated for the final condition on $u$.

According to the extension, the costate function

$$
\tilde{\lambda}=\left(\begin{array}{c}
\lambda \\
\lambda_{u}
\end{array}\right)
$$

is introduced, with $\lambda \in R^{n}$ the same as the original case, and $\lambda_{u} \in R$. The Hamiltonian function becomes

$$
\tilde{H}(z, \tilde{\lambda}, v, t)=\tilde{\alpha}^{T} z+\tilde{\lambda}^{T}(\Phi(z)+\Gamma v)=\tilde{F}(z, \tilde{\lambda})+\tilde{G}(\tilde{\lambda}) v
$$


with

$$
\begin{aligned}
\tilde{F}(z, \tilde{\lambda}) & =\tilde{\alpha}^{T} z+\tilde{\lambda}^{T} \Phi(z) \\
\tilde{G}(\tilde{\lambda}) & =\tilde{\lambda}^{T} \Gamma
\end{aligned}
$$

Expressions of the original formulation can be put in evidence in (84) and (85), observing that

$$
\begin{aligned}
\tilde{F}(z, \tilde{\lambda}) & =\alpha^{T} x+c u+\lambda^{T}(f(x)+g(x) u)= \\
& =H(x, \lambda, u) \\
\tilde{G}(z, \tilde{\lambda}) & =\lambda_{u}
\end{aligned}
$$

The necessary condition for the extended costate becomes

$$
\begin{aligned}
\dot{\tilde{\lambda}}^{T} & =-\frac{\partial \tilde{H}}{\partial z}=\tilde{\alpha}^{T}+\tilde{\lambda}^{T} \frac{\partial \Phi}{\partial z}+\tilde{\lambda}^{T} \frac{\partial \Gamma}{\partial z}= \\
& =-\tilde{\alpha}^{T}-\tilde{\lambda}^{T}\left(\frac{\partial \Phi}{\partial x} \frac{\partial \Phi}{\partial u}\right)-\tilde{\lambda}^{T}\left(\frac{\partial \Gamma}{\partial x} \frac{\partial \Gamma}{\partial u}\right)= \\
& =-\tilde{\alpha}^{T}-\tilde{\lambda}^{T}\left(\begin{array}{rr}
\frac{\partial f}{\partial x}+\frac{\partial g}{\partial x} u g \\
0 & 0
\end{array}\right)-\tilde{\lambda}^{T}\left(\begin{array}{ll}
0 & 0 \\
0 & 0
\end{array}\right)= \\
& =-\left(\alpha^{T}+\lambda^{T}\left(\frac{\partial f}{\partial x}+\frac{\partial g}{\partial x} u\right) c+\lambda^{T} g\right)
\end{aligned}
$$

in which the two blocks

$$
\begin{aligned}
\dot{\lambda} & =-\alpha-\left(\frac{\partial f}{\partial x}+\frac{\partial g}{\partial x} u\right)^{T} \lambda \\
\dot{\lambda}_{u} & =c+\lambda^{T} g
\end{aligned}
$$

can be evidenced, where (89) is the same as (27) and (90) corresponds to (14). Since the new extended problem does not have any constraint on the new input $v$, the necessary condition

$$
\frac{\partial \tilde{H}(z, \tilde{\lambda}, v, t)}{\partial v}=0
$$

can be considered, yielding to

$$
\tilde{G}(\tilde{\lambda}))=\lambda_{u}(t)=0
$$

Having assumed verified all the conditions of the original problem, on the singular arc one has, for (22), (90) and (92),

$$
\dot{\lambda}_{u}=\tilde{G}^{(1)}(x, \lambda)=G^{(0)}(x, \lambda)=0
$$

and then

$$
\frac{d^{k} \lambda_{u}}{d t^{k}}=\tilde{G}^{(k)}(x, \lambda)=G^{(k-1)}(x, \lambda)=0 \quad \forall k=1,2, \ldots
$$

holds in the time interval $t \in\left[t_{1}, t_{2}\right]$, while for $t \in\left[t_{0}, t_{1}\right] \cup\left[t_{2}, t_{f}\right] \lambda_{u}(t)$ is constant with all the time derivatives equal to zero. On these basis it is possible to state the following 
Proposition 5. For the extended dynamics, the presence of a singular solution is guaranteed in the same time interval as in the solution of the initial problem.

Thanks to Proposition 5, from the expressions in (93) and (94), and under the hypothesis (71) on the solution of the original problem, for the conditions on the existence of the singular solution for the extended dynamics, one has

$$
\tilde{G}^{(k)}(z, \tilde{\lambda})=0, \quad \text { for } k \in[0, n-1]
$$

independent from $u$.

For the $n$-th derivative, one has

$$
\tilde{G}^{(n)}(z, \tilde{\lambda})=G^{(n-1)}(x, \lambda, u)=0
$$

with $G^{(n-1)}(x, \lambda, u)$ in (72). For the extension, the control $v$ does not appear yet and then the subsequent derivative must be performed to compute the singular control.

$$
\begin{aligned}
\tilde{G}^{(n+1)}(z, \tilde{\lambda})= & G^{(n)}(x, \lambda, u)= \\
= & \frac{\partial G^{(n-1)}(x, \lambda, u)}{\partial x}(f+g u)+ \\
& -\left.\frac{\partial G^{(n-1)}(x, \lambda, u)}{\partial \lambda} \frac{\partial H(x, \lambda, u)}{\partial x}\right|^{T}+ \\
& +\frac{\partial G^{(n-1)}(x, \lambda, u)}{\partial u} v
\end{aligned}
$$

Performing the computation, thanks to the form of the compact expression (72), one obtains

$$
\frac{\partial G^{(n-1)}(x, \lambda, u)}{\partial u}=N(x, \lambda)
$$

This result proves the following

Proposition 6. If the singular control $u_{s}$ can be computed, as in (73), for the non singularity of the coefficient $N(x, \lambda)$, the same holds for the control $v$ of the extended problem.

Moreover

Proposition 7. The set of equations

$$
\begin{aligned}
\tilde{G}^{(0)}(z, \tilde{\lambda}) & =\lambda_{u}=0 \\
\tilde{G}^{(1)}(z, \tilde{\lambda}) & =G^{(0)}(x, \lambda)=\lambda^{T} g(x)+c=0 \\
\tilde{G}^{(2)}(z, \tilde{\lambda}) & =G^{(1)}(x, \lambda)=\lambda^{T} a d_{f} g(x)-\alpha^{T} g(x)=0 \\
\ldots & \ldots \\
\tilde{G}^{(n-1)}(z, \tilde{\lambda}) & =G^{(n-2)}(x, \lambda)=\lambda^{T} a d_{f}^{n-2} g(x)+h_{n-2}(x)=0 \\
\tilde{G}^{(n)}(z, \tilde{\lambda}) & =G^{(n-1)}(x, \lambda, u)=M(x, \lambda)+N(x, \lambda) u=0
\end{aligned}
$$

can be used to compute $\lambda=\lambda(x, u)$ without integrating the system (27). 
Proof. For the proof, the explicit expression of $M(x, \lambda)$ and $N(x, \lambda)$ should be put in evidence to show their relationship with $\lambda$. Making also use of (30) for reference, it can be observed that the coefficients of $\lambda$ in the terms $G^{(i)}(x, \lambda)$, for $i=0, \ldots, n-2$ are $a d_{f}^{i} g(x)$ (Proposition 1). For $G^{(n-1)}(x, \lambda, u)$, the terms in the coefficient of $\lambda$ contain $a d_{f}^{n-1} g(x)$ along with other Lie Brackets of order lower of $n$ contained in the non singular distribution

$$
D=\operatorname{span}\left\{g, a d_{f} g, \ldots, a d_{f}^{n-1} g\right\}(x)
$$

so that (99) can be always inverted with respect to $\lambda$, getting

$$
\lambda=\lambda(x, u)
$$

which can be used in the remaining part of the procedure to solve the optimal control problem, while for $\lambda_{u}$ (92) holds.

The correspondence between the original optimal solution in (20), whose expression is (73), and the optimal solution $v$ for the extended control problem can be easily stated. In fact, $v=0$ outside the singular part of the solution corresponds to $u$ constant, whose value is 0 or $u_{M A X}$, defined by means of the boundary conditions on the state component $u$ in $z$. For the singular arc, comparing the expressions of $G^{(n-1)}(x, \lambda, u)$ in (72), from which $u_{s}$ is computed, with

$$
G^{(n)}(x, \lambda, u, v)=\dot{M}(x, \lambda)+\dot{N}(x, \lambda) u+N(x, \lambda) v
$$

from which

$$
v_{s}=-\frac{\dot{M}(x, \lambda)+\dot{N}(x, \lambda) u_{s}}{N(x, \lambda)}
$$

is obtained, it is possible to verify, since $\dot{u}_{s}=v_{s}$, that the optimal solution for the state component $u$ of the extended problem coincides with the optimal control $u_{s}$, solution of the original problem. In fact

$$
\begin{aligned}
\dot{u}_{s} & =\frac{d}{d t}\left(-\frac{M(x, \lambda)}{N(x, \lambda)}\right)= \\
& =-\frac{\dot{M}(x, \lambda) N(x, \lambda)-\dot{N}(x, \lambda) M(x, \lambda)}{N^{2}(x, \lambda)}= \\
& =-\frac{\dot{M}(x, \lambda)-\dot{N}(x, \lambda) \frac{M(x, \lambda)}{N(x, \lambda)}}{N(x, \lambda)}= \\
& =-\frac{\dot{M}(x, \lambda)-\dot{N}(x, \lambda) u_{s}}{N(x, \lambda)}=v_{s}
\end{aligned}
$$

once compared with (103). 


\subsection{The example of a SIR model with no immunization}

An example is here adopted to show explicitly the procedure which allows to compute the costate $\lambda$ as a function of the state $x$ and the control $u$, so that the extended input $v$ over the singular manifold, can be expressed without the necessity of the integration of the differential system for $\lambda$.

The dynamics considered is the same as in Subsection 3.3, slightly modified in order to make it loose the particular structure (33).

The modified dynamics is of the form (1) with $x=(S I R)^{T}$,

$$
f(x)=\left(\begin{array}{c}
-\beta S I+\rho R+\mu \\
\beta S I-\gamma I \\
\gamma I-\rho R
\end{array}\right), \quad g(x)=\left(\begin{array}{c}
-S \\
0 \\
0
\end{array}\right)
$$

where the change w.r.t. Subsection 3.3 is the addition of the term $\rho R$ representing the rate of recovered which can re-contract the virus, moving from $R$ to $S$.

In the cost function, the term $L$ is as in (53), of the form (25). The Hamiltonian function is as in (12), with $\alpha=\left(\alpha_{1} \alpha_{2} \alpha_{3}\right)^{T}$ so that the functions $G^{(0)}(x, \lambda)$, $G^{(1)}(x, \lambda)$ and $G^{(2)}(x, \lambda, u)$ have the expressions (28), (29) and (30) respectively. The differential system for $\lambda$ arising from the necessary conditions is

$$
\begin{aligned}
& \dot{\lambda}_{1}=-\left(\alpha_{1}-\beta I \lambda_{1}+\beta I \lambda_{2}-\gamma \lambda_{2}-\lambda_{1} u\right) \\
& \dot{\lambda}_{2}=-\left(\alpha_{2}-\beta S \lambda_{1}+\beta S \lambda_{2}+\gamma \lambda_{3}\right) \\
& \dot{\lambda}_{3}=-\left(\alpha_{3}+\rho \lambda_{1}-\rho \lambda_{3}\right)
\end{aligned}
$$

which should be solved, along with (1), under mixed boundary conditions.

In this case, if the extension (74) is introduced, the costate $\lambda$ can be computed as a function of $x$ and $u$ directly solving the system (28)-(29)-(30).

For the present case, one gets

$$
\begin{gathered}
a d_{f} g(x)=\left(\begin{array}{c}
\rho R+\mu \\
-\beta S I \\
0
\end{array}\right), \quad a d_{g} a d_{f} g(x)=\left(\begin{array}{c}
\rho R+\mu \\
-\beta S I \\
0
\end{array}\right) \\
L_{g} g(x)=\left(\begin{array}{l}
S \\
0 \\
0
\end{array}\right), \quad L_{f} g(x)=\left(\begin{array}{c}
\beta S I-\rho R-\mu \\
0 \\
0
\end{array}\right) \\
a d_{f}^{2} g(x)=\left(\begin{array}{c}
\rho \gamma I-\rho^{2} R+\rho \beta I R+\mu \beta I-\beta^{2} S^{2} I \\
\beta^{2} S I^{2}-2 \rho \beta I R-2 \beta I \\
-\beta \gamma S I
\end{array}\right)
\end{gathered}
$$

and the system to be solved becomes

$$
\lambda^{T} C(x, u)=D(x, u)
$$

with

$$
C(x, u)=\left(g_{a} g d_{f} a d_{f}^{2} g+a d_{g} a d_{f} g u\right)=
$$




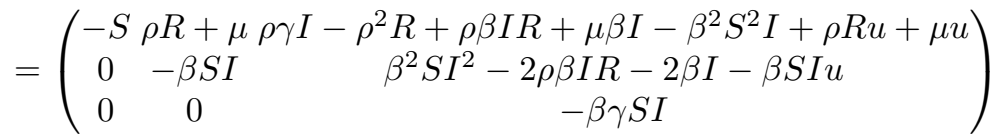

$$
\begin{aligned}
& D(x, u)=\left(-c \alpha^{T} g \alpha^{T} L_{g} g u+\alpha^{T}\left(L_{f} g+a d_{f} g\right)\right)
\end{aligned}
$$

It can be verified that the controllability condition is satisfied once $S \neq 0$ and $I \neq 0$. Under the same conditions,

$$
\lambda^{T}(x, u)=D(x, u) C^{-1}(x, u)
$$

can be computed, since, as it is easy to verify by visual inspection, the determinant $\Delta$ of the matrix $C(x, u)$ is $\Delta=-\beta^{2} \gamma S^{3} I^{2} \neq 0$ and then $C(x, u)$ is invertible.

\section{Conclusions}

Sufficient conditions under which the solution of a singular optimal control problem can be directly expressed as a state feedback law are provided, allowing its computation by means of a simple forward integration of the system dynamics only.

The necessity of a particular structure for the systems for satisfying the conditions required for the implementation of the proposed procedure has been overcome, introducing a dynamic extension on the input. The effectiveness of the approach is shown for the case in which an order one integrator is sufficient, but the proof of the success of the proposed approach for a generic order can be obtained, for example, by iterative application of the one dimensional single step.

\section{Acknowledgements}

This work was supported by Sapienza University of Rome, Grants No. 191/2016 and No. RP11715C82440B12.

\section{References}

1. M. Athans and P.L. Falb. Optimal Control. McGraw-Hill, Inc., New York, 1996.

2. R.F. Hartl, S.P. Sethi, and R.G. Vickson. A survey of the maximum principles for optimal control problems with state constraints. Society for Industrial and Applied Mathematics, 37:181-218, 1995.

3. C.D. Johnson and J.E. Gibson. Singular solutions in problems of optimal control. IEEE Trans. on Automatic Control, 8(1):4-15, 1963.

4. A.E. Bryson and Y.C. Ho. Applied optimal control: optimization, estimation, and control. 1969.

5. G. Vossen. Switching time optimization for bang-bang and singular controls. Journal of Optimization Theory and Applications, 144, 2010. 
6. G. Fraser-Andrews. Finding candidate singular optimal controls: a state of art survey. Journal of Optimization Theory and Applications, 60, 1989.

7. P. Di Giamberardino and D. Iacoviello. Optimal control of SIR epidemic model with state dependent switching cost index. Biomedical Signal Processing and Control, 31, 2017.

8. E.A. Bakare, A. Nwagwo, and E. Danso-Addo. Optimal control analyis of an sir epidemic model with constant recruitment. International Journal of Applied Mathematical Research, 3, 2014.

9. H. Behncke. Optimal control of deterministic epidemics. Optimal control applications and methods, 21, 2000.

10. P. Di Giamberardino, L. Compagnucci, C. De Giorgi, and D. Iacoviello. Modeling the effects of prevention and early diagnosis on hiv/aids infection diffusion. IEEE Transactions on Systems, Man and Cybernetics: Systems, 2018.

11. U. Ledzewicz and E. Schattler. On optimal singular controls for a general SIRmodel with vaccination and treatment. Discrete and continuous dynamical systems, 2011.

12. U. Ledzewicz, M. Aghaee, and H. Schattler. Optimal control for a sir epidemiological model withtime-varying population. 2016 IEEE Conference on Control Applications, 2016.

13. P. Di Giamberardino and D. Iacoviello. State feedback optimal control with singular solution for a class of nonlinear dynamics. In Proceedings of the 15th International Conference on Informatics in Control, Automation and Robotics (ICINCO 2018), 1, 2018. 